\title{
Clinical Characteristics of Tracheobronchopathia Osteochondroplastica
}

\author{
Ting Luo, Hui Zhou, and Jie Meng
}

\begin{abstract}
BACKGROUND: Tracheobronchopathia osteochondroplastica (TO) consists of benign lesions of tracheal and bronchial mucosa with multiple nodular hyperplasia of bone or cartilage protruding into the lumen. METHODS: We diagnosed 73 subjects with TO with the use of bronchoscopy in the Department of Respiratory and Critical Care Medicine of Xiangya Hospital between January 2000 and April 2017. Clinical manifestations, radiographic characteristics, bronchoscopic manifestations, histopathological findings, and treatments were analyzed retrospectively. RESULTS: Subjects included 30 women and 43 men (mean age, $52.8 \pm 11.7$ y). Twenty-seven subjects were diagnosed with other diseases, including tuberculosis in 11 subjects, carcinoma in 13, bronchiectasis in 2 , and pulmonary hamartomas in 1 subject. The most common symptom was cough $(n=34)$. Other initial manifestations included hemoptysis $(n=17)$, expectoration $(n=15)$, and chest pain $(n=8)$. Of those who underwent a computed tomography scan in our hospital, 28 of 32 subjects had noted calcification of the tracheal wall, with 4 of 32 subjects appearing normal. Bronchoscopy revealed typical accumulation of diffuse cartilaginous and osseous nodules of TO, and 9 had observable tracheal stenosis. Histopathologic results included the presence of chronic inflammation $(n=47)$, ossification $(n=38)$, and cartilage formation $(n=10)$. Most subjects received symptomatic treatment, although 3 subjects with severe airway obstruction received bronchoscopic treatment. CONCLUSION: The results indicated that TO lacks clinical specificity, which means that special therapy and bronchoscopy with histopathological and radiographic assistance is important for its diagnosis. The treating physicians' awareness about this condition is also important to its diagnosis and management. Key words: retrospective analysis; tracheobronchopathia osteochondroplastica; bronchoscopy; clinical manifestation. [Respir Care 2019;64(2):196-200. (C) 2019 Daedalus Enterprises]
\end{abstract}

\section{Introduction}

Tracheobronchopathia osteochondroplastica (TO) is a relatively rare benign tracheal disease, which is character-

\footnotetext{
Drs Meng and Luo are affiliated with the Department of Respiratory and Critical Care Medicine, Xiangya Hospital, Central South University, Changsha, Hunan, China. Dr Zhou is affiliated with the Department of Radiology, Xiangya Hospital, Central South University, Changsha, Hunan, China.

Dr Meng reports a relationship with the National Natural Science Foundation of China. Drs Meng and Zhou report relationships with the Natural Science Foundation of Hunan Province.
}

Correspondence: Jie Meng, Department of Respiratory and Critical Care Medicine, Xiangya Hospital, Central South University, Changsha, Hunan, China. E-mail: mengjie@csu.edu.cn.

DOI: $10.4187 /$ respcare.05867 ized by the accumulation of diffuse cartilaginous and osseous nodules that protrude into the anterolateral walls of the trachea and bronchus. ${ }^{1}$ The etiology and pathogenesis of TO remain unknown. It was first described by Wilks in the 19th century. ${ }^{2}$ In 1910, Aschoff named it tracheobronchopathia osteochondroplastica. ${ }^{3}$ Few clinical papers about TO have been published. The clinical manifestations of the disease are variable, and its symptoms lack specificity. Bronchoscopy remains the accepted standard for the recognition of TO, although other diagnostic methods including chest computed tomography (CT) and biopsy are also widely used. ${ }^{4}$ To date, there is no specific treatment for TO. In some cases, bronchoscopic radiofrequency and argon plasma coagulation therapy have been effective palliative treatments. ${ }^{5,6}$ By April 2017, approximately 500 cases had been reported globally. ${ }^{7}$ With the rapid development of bronchoscopy, TO has become relatively easy to diagnose, and reports of this disease have increased in recent years. In this study, 
we aim to provide a better description of TO to further assist understanding of this disease.

\section{Methods}

Among 67,208 patients who had undergone bronchoscopies (Olympus BF-P260F, Olympus, Japan) in Xiangya Hospital between January 2000 and April 2017, 73 subjects were diagnosed with $\mathrm{TO}$ with or without histopathologic findings by 2 pulmonary physicians who had more than 5 years of experience in bronchoscopy. This study was reviewed and approved by the ethics committee of the Xiangya Hospital of Central South University. All of the subjects signed the informed consent form before they underwent bronchoscopic examination. The characteristic manifestations were described as multiple papilla-like sessile cartilaginous or osseous nodules arising from the submucosa and protruding selectively from the anterolateral wall of the tracheobronchial tree. ${ }^{8}$ The categories of subjects were classified based on criteria established in a study of a large Chinese cohort, which classified three stages of TO based on the severity of bronchoscopic findings: Stage I (early stage, also a mild grade), consisting of plaque-like infiltrations of soft, yellow-whitish lesions scattered in the lumen mucosa, accompanied by a change of mucosa hyperemia edema; Stage II (middle stage, moderate grade), which exhibits diffuse cartilaginous nodules and sessile spicules projecting into the lumen with a cobblestone or stalactite appearance; Stage III (late stage, severe grade), demonstrating deformed and rigid stenosis of the airway that may cause obstruction. ${ }^{9}$

\section{Results}

Our study group included 30 women (41.1\%) and 43 men $(58.9 \%)$. Subject age ranged from 26 to $76 \mathrm{y}$ with a mean age of $52.8 \pm 11.7 \mathrm{y}$. Twenty-seven subjects had been diagnosed with other diseases: 11 subjects were previously diagnosed with tuberculosis, 13 subjects were previously diagnosed with carcinoma (ie, small cell lung cancer in 4 subjects, pulmonary adenocarcinoma in 6 subjects, pulmonary squamous carcinoma in 1 subject, breast carcinoma in 1 subject, and esophageal cancer in 1 subject), pulmonary hamartomas in 1 subject, and 2 subjects had been previously diagnosed with bronchiectasis. Twenty-one subjects were active or former smokers.

The most common symptom was cough $(n=34)$, and other clinical manifestations included hemoptysis $(n=17)$, expectoration $(n=15)$, chest pain $(n=8)$, dyspnea $(n=7)$, and fever $(n=3)$.

Thirty-two subjects underwent chest CT in our hospital, as shown in Figure 1. Lesions and submucosal calcifications were present in 28 subjects, whereas the other 4 individuals had normal findings. Among the 28 subjects, 8

\section{QUICK LOOK}

\section{Current knowledge}

Tracheobronchopathia osteochondroplastica (TO) is a relatively rare benign tracheal disease, and its etiology and pathogenesis remains unknown. Bronchoscopy remains the accepted standard for the recognition of TO, but there is no internationally unified diagnostic standard for TO until now.

\section{What this paper contributes to our knowledge}

In this study, we aim to provide a better description of $\mathrm{TO}$ to assist in understanding this disease. TO can be diagnosed after other diseases are excluded, and its diagnostic criteria include typical bronchoscopy manifestations, computed tomography and histopathological findings.

presented with calcification and thickening of the tracheal wall, and the trachea remained smooth. Of the 20 subjects who showed a rough lumen with multiple irregular calcified nodules protruding into the lumen, 3 displayed irregular stenosis of the main bronchi accompanied by ample, densely calcified nodules.

Bronchoscopy showed 9 subjects with tracheal stenosis (Fig. 2), of which 2 had lesions confined to the bronchus, whereas the remaining 7 exhibited lesions in the trachea. Thirty-eight subjects presented with scattered or diffuse cartilaginous nodules and sessile spicules projecting into the lumen without obstruction. Based on the severity of bronchoscopic findings, subjects were classified into 3 categories: 38 of 73 subjects were classified as Stage II, which was the largest group in this study, followed by Stage I (26 subjects) and Stage III (9 subjects), respectively.

As shown in Table 1, the histopathologic results of 71 subjects were obtained. Histopathologic findings (Fig. $3)$ included chronic inflammation $(n=47)$, ossification $(n=38)$, calcification $(n=9)$, and cartilage formulation $(n=10)$ in the bronchial submucosa. Five subjects had squamous metaplasia and mild atypical hyperplasia.

In this study, 3 subjects who presented with severe airway obstruction and debilitating symptoms received repeated bronchoscopic treatment, including argon plasma coagulation, radiofrequency, and microwave therapy. Clinical symptoms (such as cough and dyspnea) were relieved, and their quality of living was improved after therapy. The other subjects received symptomatic treatments, such as anti-infection treatment, spasmolysis, hemostatics, antitussives, and expectorants.

During follow-up, 5 subjects died of lung cancer ( 4 of small cell lung cancer, 1 of pulmonary adenocarcinoma). 

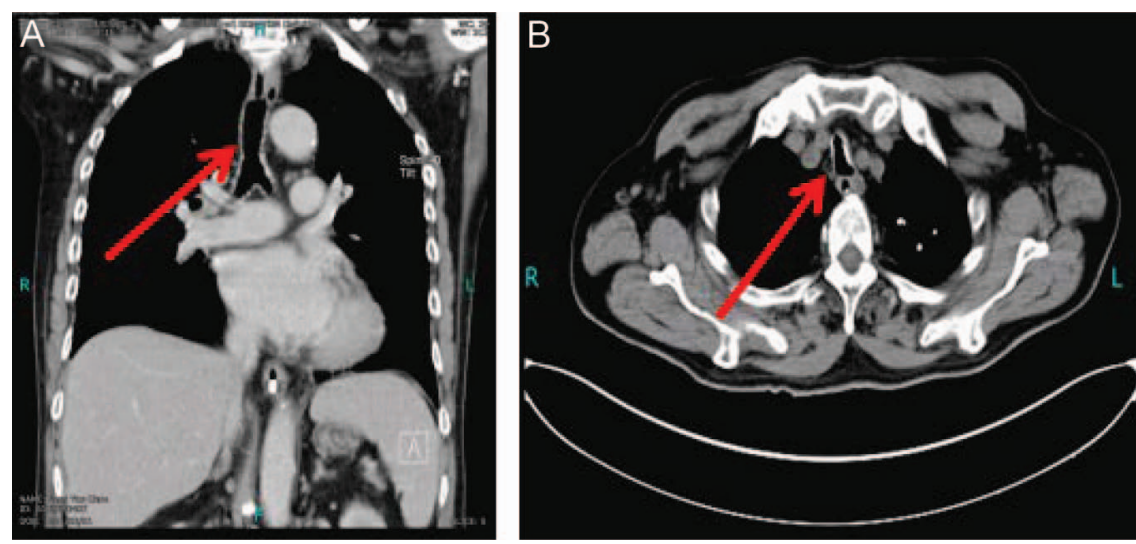

Fig. 1. Chest computed tomography showing diffuse irregular thickening and calcified anterolateral nodules protruding into the tracheal lumen (arrows).
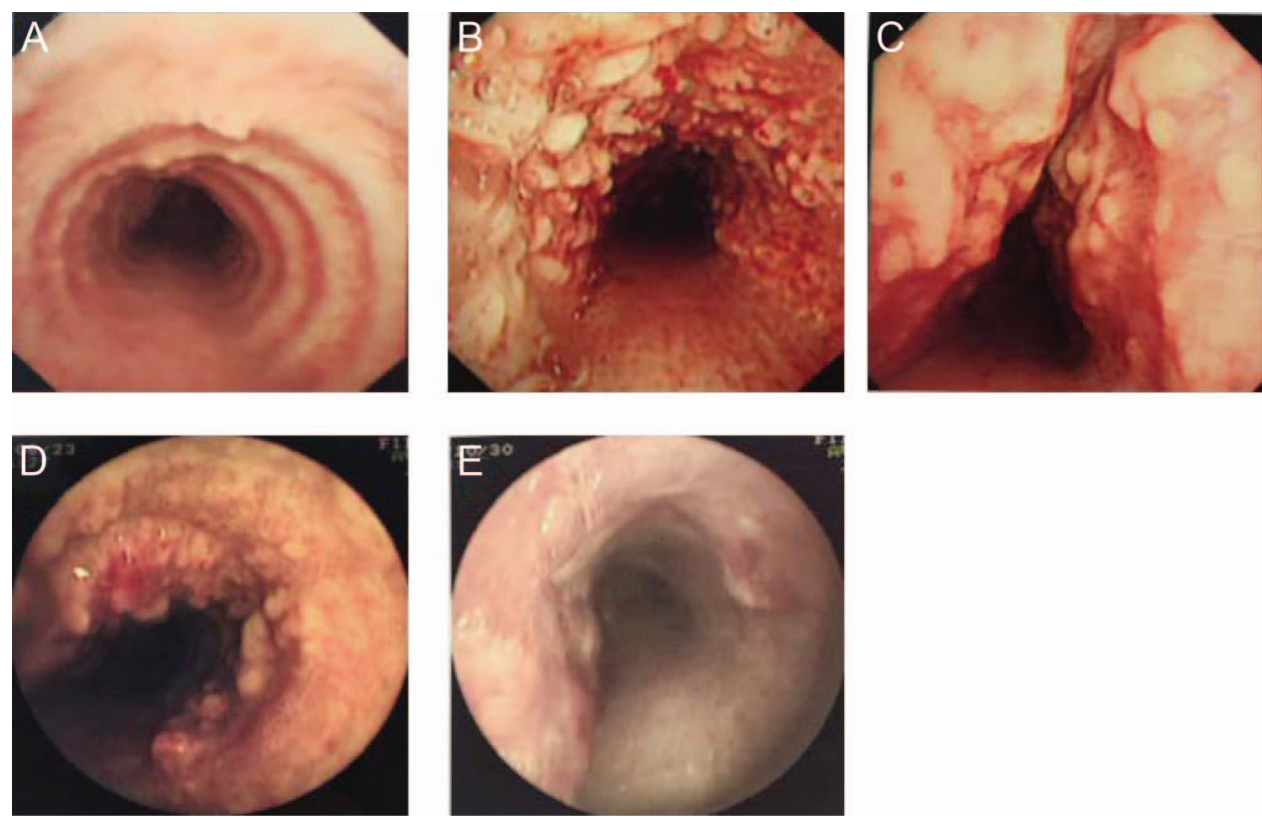

Fig. 2. The important characteristic in tracheobronchopathia osteochondroplastica is sparing of the posterior wall. (A) Stage I: Scattered lesions overlying the mucosa of the lumen. (B) Stage II: Numerous dispersed or diffuse nodules of bone and cartilage projecting into the lumen. (C and D) Stage III: The airway is deformed, narrow, and rigid. (E) After treatment with argon plasma coagulation, the number of diffuse cartilaginous nodules decreased.

The other subjects had chronic or recurrent cough and expectoration.

Table 1. Histopathologic Findings From Tracheobronchial Biopsies

\begin{tabular}{lc}
\hline \hline \multicolumn{1}{c}{ Finding } & Subjects, $n(\%)$ \\
\hline Chronic inflammation & $47(66.2)$ \\
Squamous metaplasia & $5(7.0)$ \\
Ossification & $38(53.5)$ \\
Cartilage formulation & $10(14.1)$ \\
Calcification & $9(12.7)$ \\
& \\
$n=71$ subjects. & \\
\hline
\end{tabular}

\section{Discussion}

Tracheobronchopathia osteochondroplastica is a rare benign airway disease, with an incidence ranging from 1:400 $(0.25 \%)$ to $3: 1,000(0.30 \%)$ in autopsies and $1: 125(0.80 \%)$ to $1: 10,000(0.01 \%)$ via bronchoscopy, ${ }^{10}$ although accurate incidence of TO remains unclear. TO has become relatively easy to diagnose with the widespread use of bronchoscopy. However, there are still a number of misdiagnosed cases, so a better understanding of the disease is important.

The incidence of TO observed via bronchoscopy is about $0.1 \%$ in our result. The mean age of our TO subjects was 

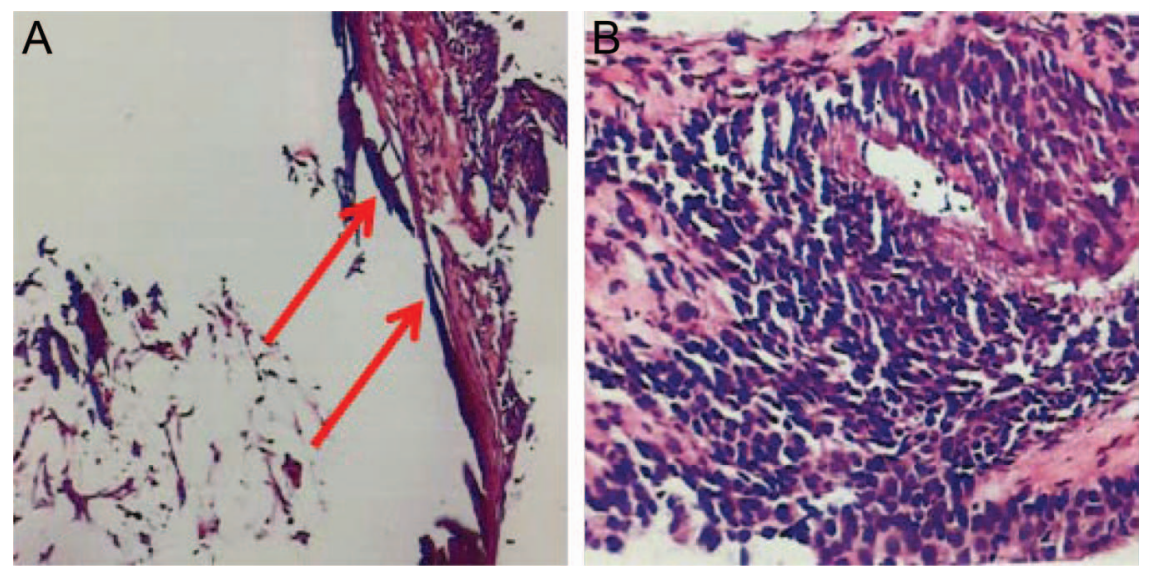

Fig. 3. Histopathological findings of tracheobronchopathia osteochondroplastica illustrate submucosal ossification and cartilage formation (arrows), accompanied by a number of inflammatory cells.

around $52 \mathrm{y}$, which was similar to previous findings. ${ }^{11}$ The incidence of TO in men was slightly higher than that in women. In our data, the youngest subject was 26 years old, and the oldest was 76 years old. However the youngest case reported previously was a 9-year-old girl. ${ }^{12} \mathrm{We}$ found no family history of TO in our subjects.

The etiology and pathophysiology of TO remains unknown. The origin of the lesions seen in TO may favor metaplasia of submucosal connective tissue or ecchondrosis and exostosis of tracheal cartilage originating from a tracheal ring. ${ }^{13-15}$ Tajima et al ${ }^{16}$ found that bone morphogenetic protein 2 could interact with the transformation of growth factor- $\beta 1$ and promote the formation of calcific foci. Some reports have proposed that etiologies of TO include metabolic abnormalities, degenerative processes related to aging, amyloidosis, malignancy, inheritance, silicosis, chronic irritation, and infection, but these hypotheses lack significant evidence. ${ }^{17-19}$ Some case reports indicate that patients with TO who received inhaled corticosteroids for anti-inflammation therapy had symptom relief, which suggests that slowly progressing inflammatory stimulation may lead to the damage of the normal architecture of airway. ${ }^{9}$

Clinical manifestations of TO in patients are variable and nonspecific. Some reports found that the most frequent complaint was chronic cough and hemoptysis, which were similar to the subjects in this report. ${ }^{20}$ Chest CT scans may assist in demonstrating submucosal calcified nodules, which can help in the differential diagnosis of TO. ${ }^{18}$ In contrast to TO, relapsing polychondritis, another condition with a potentially similar radiological picture, presents with thickened and deformed cartilage, without intraluminal nodules. ${ }^{21}$ Although positive finding of CT scan in our study was $87.5 \%$ (28/32), TO is easy to missed in diagnosis by radiologists. Bronchoscopy is the most common and definitive diagnostic test for this condition. ${ }^{22}$ Nodules can be seen in the lumen with bronchoscopy.
Sarcoidosis, amyloidosis, and papillomatosis could have a similar endoscopic appearance, but none of these selectively spare the posterior membranous wall. ${ }^{21}$ In some cases, howeer, biopsy is needed to distinguish between TO, tracheobronchial amyloidosis, and tuberculosis. Pathological examination of TO will show ossification and calcification.

To date, there is no internationally unified diagnostic standard regarding TO. In this study, the diagnosis of TO depended on typical manifestations of bronchoscopy combined with typical CT manifestations (ie, multiple submucosal calcified nodules sparing the posterior membranous trachea) or the results of histopathological findings, after excluding other diagnoses. At the same time, the treatment and related prognosis were further guided by the classification and manifestation of bronchoscopy.

There are no widely accepted treatments for TO. Relatively effective treatment modalities include conservative treatment, microwave therapy, argon plasma coagulation, bronchoscopy-guided excision of nodules, surgical resection, and bronchoscopic radiofrequency ablation. These treatments can improve patients' quality of life, especially those who are suffering from airway obstruction. The prognosis of TO is usually favorable, but this depends upon the extent and location of the nodular lesions. ${ }^{20}$

\section{Conclusion}

Tracheobronchopathia osteochondroplastica consists of benign lesions of the trachea and lacks a typical clinical manifestation. While the most important diagnostic tool for TO is bronchoscopy, CT imaging and biopsy can help differentiate TO from other diseases. Moreover, the treating physicians' awareness of this condition is key to its diagnosis and management. No specific treatments are recommended at present, although endoscopic therapy is some- 


\section{TRACHEOBRONCHOPATHIA OSTEOCHONDROPLASTICA}

times used to treat severe airway obstruction and improve patient quality of life.

\section{REFERENCES}

1. Leske V, Lazor R, Coetmeur D, Crestani B, Chatte G, Cordier JF. Tracheobronchopathia osteochondroplastica: a study of 41 patients. Medicine 2001;80(6):378-390.

2. Silveira MGM, Castellano M, Fuzi CE, Coletta E, Spinosa GN. Tracheobronchopathia osteochondroplastica. J Bras Pneumol 2017; 43(2):151-153.

3. Karlikaya C, Yuksel M, Kilicli S, Candan L. Tracheobronchopathia osteochondroplastica. Respirology 2000;5(4):377-380.

4. Abu-Hijleh M, Lee D, Braman SS. Tracheobronchopathia osteochondroplastica: a rare large airway disorder. Lung 2008;186(6): 353-359.

5. Meyer CN, Dossing M, Broholm H. Tracheobronchopathia osteochondroplastica. Respir Med 1997;91(8):499-502.

6. Li YY, Hu CP, Yang HZ, Yang HP, Qu SJ, Zhou YZ. [The diagnostic value of flexible bronchoscopy in tracheobronchopathia osteochondroplastica]. Zhonghua Jie He He Hu Xi Za Zhi 2009;32(7): 489-492.

7. Wang N, Long F, Jiang S. Tracheobronchopathia osteochondroplastica: two cases reports and review of literature. Medicine 2016; 95(19):e3396.

8. Barthwal MS, Chatterji RS, Mehta A. Tracheobronchopathia osteochondroplastica. Indian J Chest Dis Allied Sci 2004;46(1):43-46.

9. Zhu Y, Wu N, Huang HD, Dong YC, Sun QY, Zhang W, et al. A clinical study of tracheobronchopathia osteochondroplastica: findings from a large Chinese cohort. PloS One 2014;9(7):e102068.

10. Prakash UB. Tracheobronchopathia osteochondroplastica. Semin Respir Crit Care Med 2002;23(2):167-175.
11. Luo S, Wu L, Zhou J, Xu S, Yang Q, Li Y, et al. Tracheobronchopathia osteochondroplastica: two cases and a review of the literature. Int J Clin Exp Pathol 2015;8(8):9681-9686.

12. Simsek PO, Ozcelik U, Demirkazik F, Unal OF, Orhan D, Aslan AT, Dogru, D. Tracheobronchopathia osteochondroplastica in a 9-yearold girl. Pediatr Pulmonol 2006;41(1):95-97.

13. Martin CJ. Tracheobronchopathia osteochondroplastica. Arch Otolaryngol 1960) 1974;100(4):290-293.

14. Jindal S, Nath A, Neyaz Z, Jaiswal S. Tracheobronchopathia osteochondroplastica: a rare or an overlooked entity? J Radiol Case Rep 2013;7(3):16-25.

15. Pounder D, Pieterse A. Tracheopathia osteoplastica: a study of the minimal lesion. J Pathol 1982;138(3):235-239.

16. Tajima K, Yamakawa M, Katagiri T, Sasaki H. Immunohistochemical detection of bone morphogenetic protein-2 and transforming growth factor beta-1 in tracheopathia osteochondroplastica. Virchows Archiv 1997;431(5):359-363.

17. Harma RA, Suurkari S. Tracheopathia chondro-osteoplastica: a clinical study of thirty cases. Acta Otolaryngol 1977;84(1-2):118-123.

18. Hodges MK, Israel E. Tracheobronchopathia osteochondroplastica presenting as right middle lobe collapse: diagnosis by bronchoscopy and computerized tomography. Chest 1988;94(4):842-844.

19. Wozniak MS, Buchwald J, Stockdale I, Doniec Z. Tracheobronchopathia osteochondroplastica: A 61-year-old female with middle lobe syndrome. Adv Respir Med 2017;85(3):158-160.

20. Ulasli SS, Kupeli E. Tracheobronchopathia osteochondroplastica: a review of the literature. Clin Respir J 2015;9(4):386-391.

21. Devaraja K, Sagar P, Chirom AS. Tracheobronchopathia osteochondroplastica: awareness is the key for diagnosis and management. BMJ Case Rep 2017;2017.

22. Sun J, Xie L, Su X, Zhang X. Tracheobronchopathia osteochondroplastica: case report and literature review. Respir Med Case Rep 2015;15:14-17. 